University of Wollongong

Research Online

Faculty of Engineering and Information

Faculty of Engineering and Information

Sciences - Papers: Part A

Sciences

$1-1-2008$

\title{
Thermal and electrical characterization of silicon photomultiplier
}

M Petasecca

Italian National Institute of Nuclear Physics, marcop@uow.edu.au

B Alpat

Italian National Institute of Nuclear Physics

G Ambrosi

Italian National Institute of Nuclear Physics

P Azzarello

Italian National Institute Of Nuclear Physics

R Battiston

Italian National Institute Of Nuclear Physics

See next page for additional authors

Follow this and additional works at: https://ro.uow.edu.au/eispapers

Part of the Engineering Commons, and the Science and Technology Studies Commons

Research Online is the open access institutional repository for the University of Wollongong. For further information contact the UOW Library: research-pubs@uow.edu.au 


\title{
Thermal and electrical characterization of silicon photomultiplier
}

\begin{abstract}
Detection of low levels of light is one of the key aspects in medical and space applications. Silicon photomultiplier, a novel type of avalanche photodetector which operates in Geiger mode, shows promising results and offer superior design options. The performance characteristics of the SiPM realized in FBK-irst are studied and presented in this paper. The leakage current, dark rate and internal gain are characterized as a function of temperature. The investigation has been carried out in the framework of the DASiPM Collaboration and the INFN/FBK-irst MEMS project. (C) 2008 IEEE.
\end{abstract}

\section{Keywords}

thermal, electrical, photomultiplier, characterization, silicon

Disciplines

Engineering | Science and Technology Studies

\section{Publication Details}

Petasecca, M., Alpat, B., Ambrosi, G., Azzarello, P., Battiston, R., lonica, M., Papi, A., Pignatel, G. U. \& Haino, S. (2008). Thermal and electrical characterization of silicon photomultiplier. IEEE Transactions on Nuclear Science, 55 (3), 1686-1690.

\section{Authors}

M Petasecca, B Alpat, G Ambrosi, P Azzarello, R Battiston, M lonica, A Papi, G U. Pignatel, and S Haino 


\title{
Thermal and Electrical Characterization of Silicon Photomultiplier
}

\author{
M. Petasecca, B. Alpat, G. Ambrosi, P. Azzarello, R. Battiston, M. Ionica, A. Papi, G. U. Pignatel, and S. Haino
}

\begin{abstract}
Detection of low levels of light is one of the key aspects in medical and space applications. Silicon photomultiplier, a novel type of avalanche photodetector which operates in Geiger mode, shows promising results and offer superior design options. The performance characteristics of the SiPM realized in FBK-irst are studied and presented in this paper. The leakage current, dark rate and internal gain are characterized as a function of temperature. The investigation has been carried out in the framework of the DASiPM Collaboration and the INFN/FBK-irst MEMS project.
\end{abstract}

Index Terms-Photodiode, silicon photomultiplier, thermal characteristics.

\section{INTRODUCTION}

$\mathbf{T}$ HE silicon avalanche photodetector operating in Geiger mode, known as a silicon photomultiplier ( $\mathrm{SiPM}$ ), is composed of several micro-cells, each one connected to an integrated quenching resistance and to a common electrode. In Geiger mode operation the diode is polarized at a bias voltage above the breakdown voltage [1]. In this condition, any electron-hole pair produced by thermal effect or incident light generates a current pulse with a charge amplification factor up to $10^{6}$.

The signal produced by a single micro-cell carries digital information: when hit by a single photon the sensor produces a single pulse current. However, when an array of identical microcells is hit by incident light, the amplitude of the produced signal (summed across the whole matrix) is proportional to the number of triggered micro-cells.

The main parameters which affect the performance of an SiPM sensor are: the dark current, the internal gain, and the dark rate. The noise figure of a Geiger mode photodetector can be identified with the dark rate defined as the number of avalanche current pulses produced by thermally generated electrons, mimicking the detection of single photons. The dark count rate is therefore the number of false events per second and should ideally be minimized.

\footnotetext{
Manuscript received November 26, 2007; revised February 10, 2008. This work was supported in part by the INFN/FBK-irst MEMS Project and in part by the MAPRAD Srl.

M. Petasecca is with the Italian National Institute of Nuclear Physics (INFN) sez. Perugia, Perugia 10-06100, Italy; the University of Perugia, Perugia 93-06100, Italy; and also with MAPRAD Srl, Perugia 19/I-06127, Italy (e-mail: marco.petasecca@maprad.com).

B. Alpat, G. Ambrosi, P. Azzarello, R. Battiston, M. Ionica, A. Papi, and S. Haino are with the Italian National Institute of Nuclear Physics (INFN) sez. Perugia, Perugia 10-06100, Italy.

G. U. Pignatel is with the Italian National Institute of Nuclear Physics (INFN) sez. Perugia, Perugia 10 - 06100, Italy, and also with the University of Perugia, Perugia 93-06100, Italy.

Digital Object Identifier 10.1109/TNS.2008.922220
}

The dark count rate is influenced by the following three parameters: 1) the sensitive area of the SiPM (in terms of size of the subpixel and subpixel density); 2) the bias voltage; and 3) the operating temperature [2].

In this paper, we present the results of a study focused on the variation of dark rate, leakage current, and internal gain as a function of the temperature.

\section{DEVICES AND METHODS}

The SiPM is fabricated on a p-type epitaxial layer. It consists of an array of 625 micro-cells covering an area of $1 \mathrm{~mm}^{2}$. Each micro-cell $\left(40 \times 40 \mu \mathrm{m}^{2}\right)$ is composed by a shallow $\mathrm{n}^{+}$-p junction in series with a poly-silicon quenching resistance [2], [3] (Fig. 1) whose value was estimated to be approximately $300 \mathrm{k} \Omega$ [4]. All micro-cells are connected in parallel through the aluminium layer on top of the photo-sensitive side.

The sample referenced throughout this paper comes from the second production batch produced by the FBK-irst in May 2006 and is identified by code SIT6V2PD2. Breakdown voltage and leakage current (reverse biased up to $40 \mathrm{~V}$ ) were measured to be, respectively, $34.5 \mathrm{~V}$ and $2 \mu \mathrm{A}$ at room temperature. The large number of measures and the time consuming procedure adopted to stabilize the temperature of the sample, have forced us to select only one of the more representative samples within the devices characterized at room temperature. The preliminary test was performed on several SiPM coming from the same production batch and which show off a very uniform behavior in terms of leakage current and dark rate (Fig. 2).

The sensor signal was amplified by a wide-bandwidth transimpedance amplifier (TIA) based on commercially available devices (National Semiconductor OPA656) to minimize the timing distortion. The dark pulse from a single micro-cell of an SiPM is identical to that generated by a photon pulse. To study the device characteristics it is thus important to study the dark pulse rate and its amplitude. The gain can be obtained integrating the dark pulse over its pulse duration.

The testing ground (Fig. 3) was based on the ACS climatic machine $\mathrm{CH} 160 \mathrm{C}$. The chamber, covering a temperature range of -75 up to $180{ }^{\circ} \mathrm{C}$ with an uncertainty of $\pm 0.25^{\circ} \mathrm{C}$ on a volume of 160 liters, has an automatic interface remotely controlled by a personal computer. The temperature has been monitored by three sensors integrated into the machine: two located in the chamber walls and one close to the device. The CH160C has been programmed by a dedicated software tool (WinKratos) to set the target temperature and to hold this value for two hours; after this period, an automatic data acquisition system collected the measured current and dark rate from the electrometer and the scaler, respectively. 


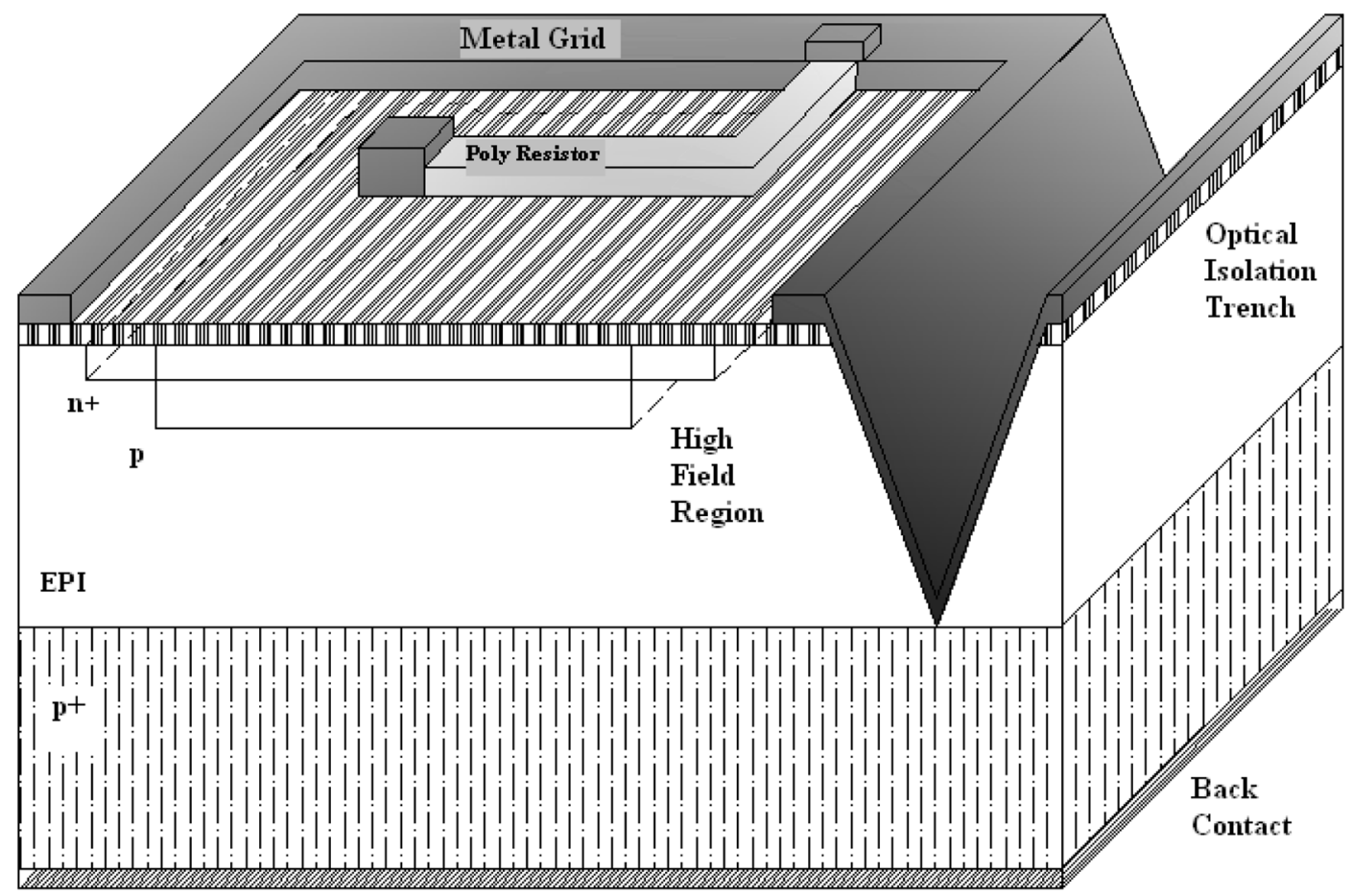

Fig. 1. SiPM border region structure.

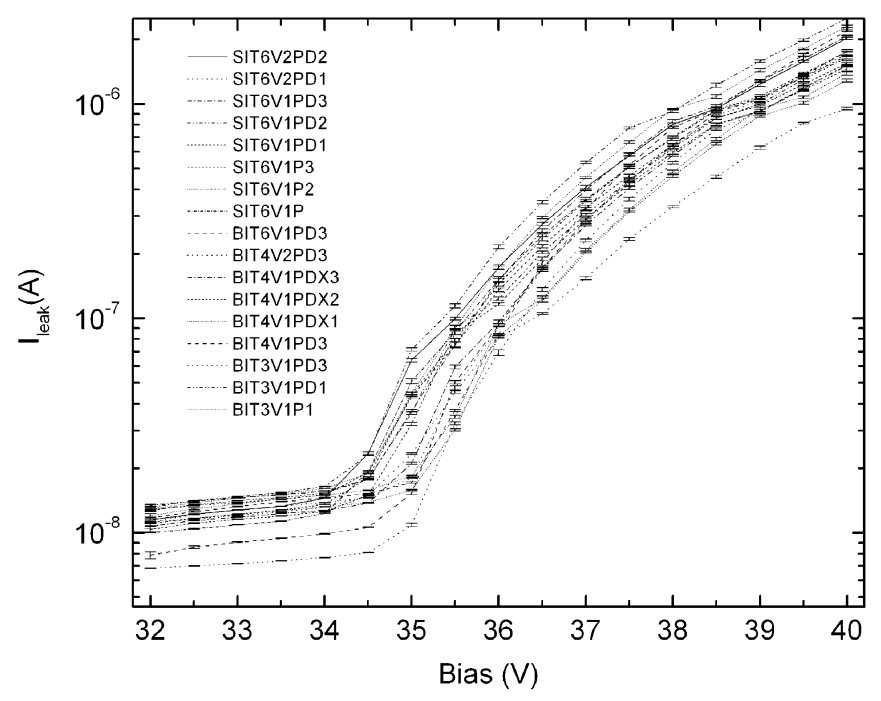

Fig. 2. SIPM dark current as a function of the bias (at $300 \mathrm{~K}$ ) for several samples from the same production batch.

\section{EXPERIMENTAL RESULTS}

\section{A. Breakdown Voltage}

Fig. 4 shows the relationship between the leakage current and bias voltage for six different temperatures. The curves are approximately linear up to the breakdown potential $\left(V_{B}\right)$, beyond which leakage current increases quadratically. This is consistent with well-known results in the literature [3]. $V_{B}$ has been estimated, for each temperature, using a semilogarithmic plot representation of the leakage current. In the plot of Fig. 4, the $V_{B}$ has been identified by the interception point of the linear

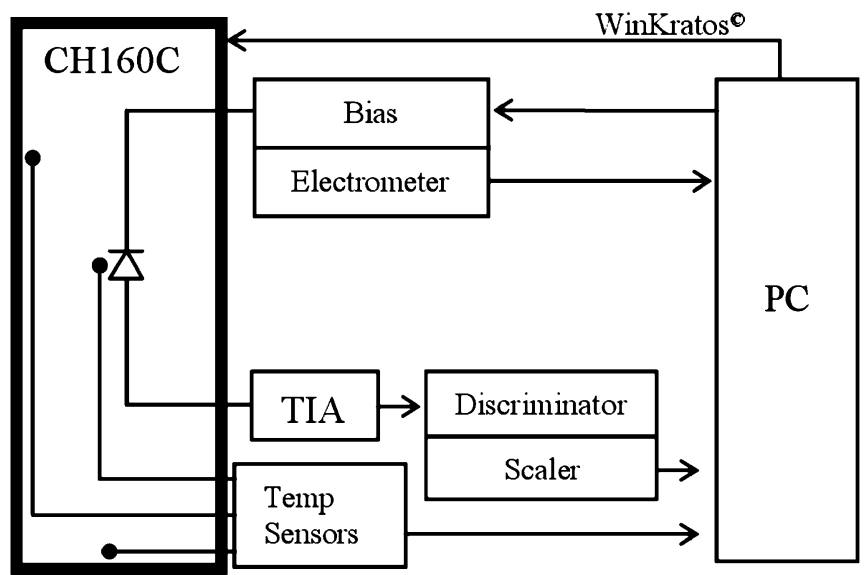

Fig. 3. Block diagram of the test bench used for the thermal characterization of SIPM.

current before the breakdown and the parabolic plot of current when SiPM works in Geiger mode.

For a pure avalanche breakdown process [5], [6], the breakdown voltage can be approximated as

$$
V_{B}=V_{B 0}\left[1+\beta\left(T-T_{0}\right)\right]
$$

where $V_{B 0}$ is the breakdown voltage at room temperature $\left(T_{0}\right)$ and $\beta$ is the linear growth constant whose value is greater than $10^{-3} \mathrm{~K}^{-1}$. For our sample, this model fits the experimental data for $\beta$ equal to $2 \pm 0.068 \cdot 10^{-3} \mathrm{~K}^{-1}$.

A qualitative explanation of the breakdown voltage increase with temperature is that hot carriers passing through the depletion layer under a high electric field, greater than $10^{6} \mathrm{~V} / \mathrm{cm}$, lose part of their energy in optical phonons through lattice scattering, 


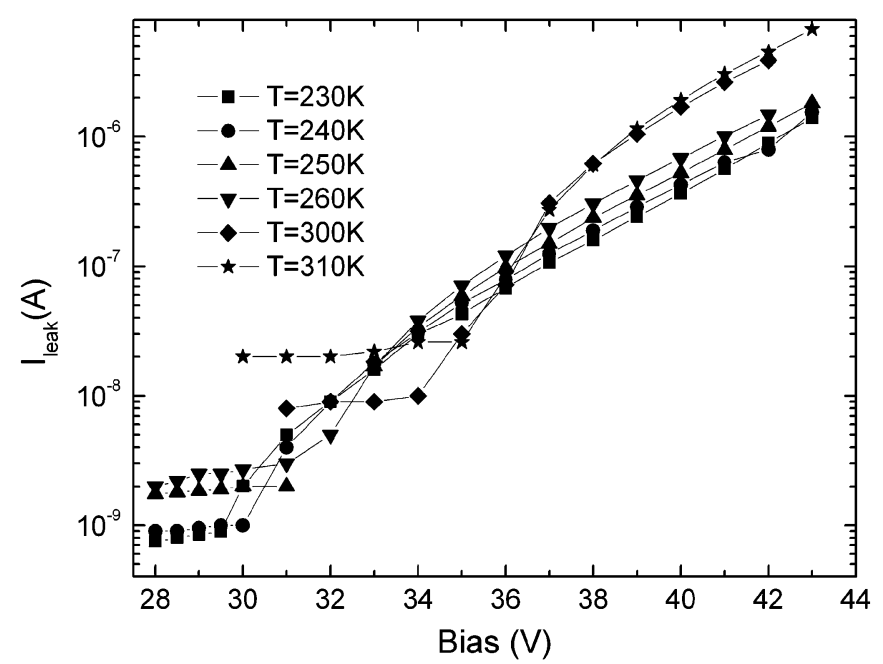

Fig. 4. SIPM dark current as a function of the bias at different operating temperatures.

resulting in a smaller ionization rate $\alpha_{\mathrm{n}, \mathrm{p}}$; hence, the carriers must overcome a greater potential difference (or a higher applied voltage) to gain sufficient energy for the generation of an electron-hole pair and generate the consequent avalanche discharge [7]. The plot in Fig. 6 shows that $V_{B}$ grows with a slope of $70.2 \pm 2.4 \mathrm{mV} / \mathrm{K}$.

\section{B. Gain}

Gain has been measured without any external light source [3]. It can be expressed as a function of the dark count rate and leakage current as

$$
G=\frac{I_{\text {leak }}}{\mathrm{dc} \cdot q}
$$

where $q$ is the electron charge, $\mathrm{dc}$ is the dark count rate, and $I_{\text {leak }}$ is the rms leakage current.

The gain of an SiPM can be determined also by the analysis of the shifting of the noise spectrum peak with the bias. This technique, proposed in several works [1], [3], [8], allows to take into account also the contribution of the after-pulsing and crosstalking between adjacent subpixels.

A comparison between the gains calculated by the spectrum shifting and with the current shows a good agreement (Fig. 5). The little variation (less than $7 \%$ for overvoltage up to $4 \mathrm{~V}$ ) between gains obtained with these two techniques means that the contribution of the crosstalk and after-pulsing to the leakage current can be consider negligible in our measurements.

Fig. 7 shows the gain as a function of the bias voltage measured for six different temperatures. The linear growth of the curve at room temperature matches results reported in a previous work [2]. In particular, gain values in the range $1.5 \cdot 10^{6}-3.5 \cdot 10^{6}$ were measured for overvoltages ranging from 0.5 to $4 \mathrm{~V}$.

It is important to note that the gain is not directly related to temperature. It decreases with increasing temperature as a result of the increase in the breakdown voltage. This is shown in Fig. 8, where the gain has been reported as a function of the bias overvoltage. The overvoltage is defined as

$$
V_{\mathrm{OV}}(T)=V_{\mathrm{BIAS}}-V_{B}(T)
$$

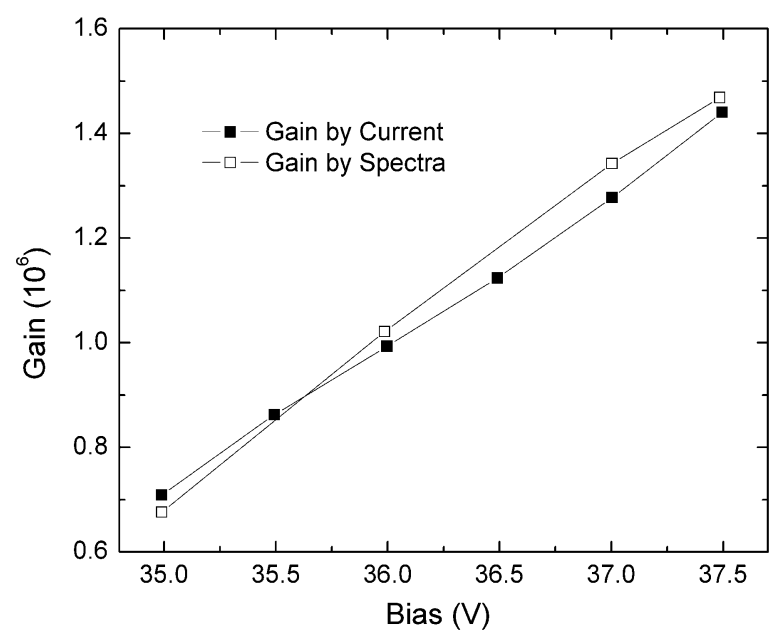

Fig. 5. Gain as a function of the bias calculated by the shifting of the dark noise spectrum and by the current measurement.

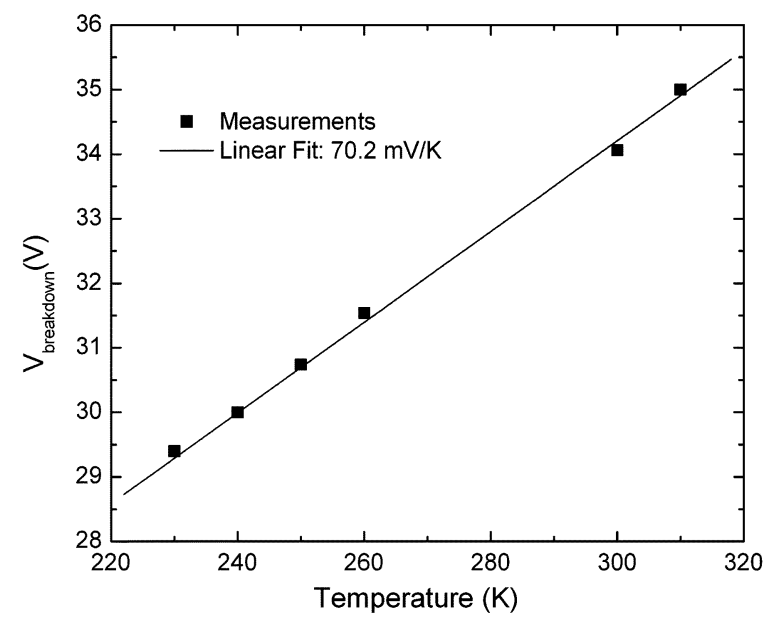

Fig. 6. Breakdown voltage as a function of the temperature.

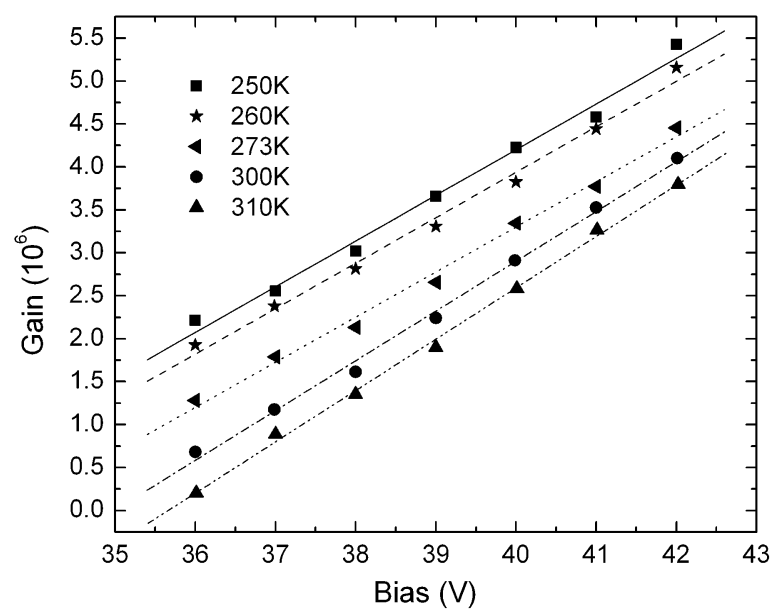

Fig. 7. Gain as a function of the bias for different operating temperatures. The gain is still linear and is independent of the temperature.

where $V_{\mathrm{BIAS}}$ is the bias voltage and $V_{B}(T)$ is the breakdown voltage measured at the operating temperature. 


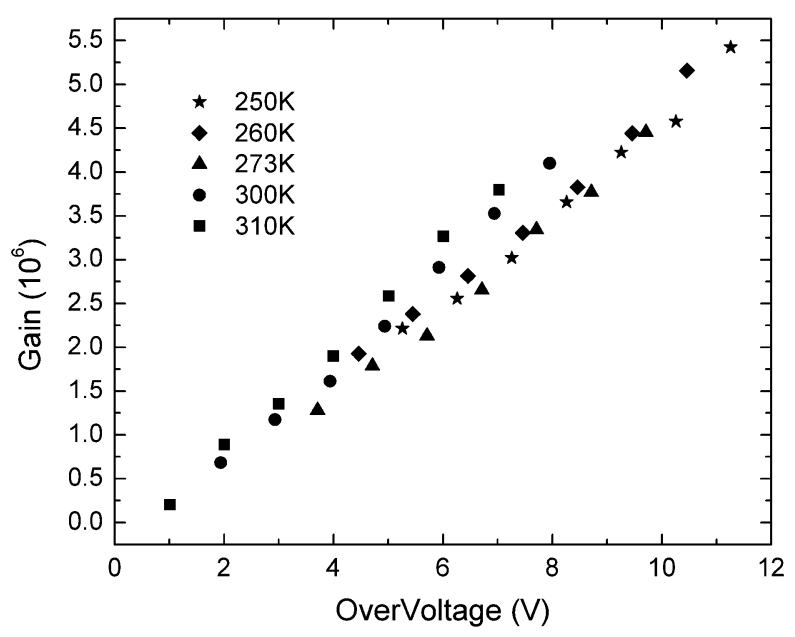

Fig. 8. Gain as a function of the overvoltage. The gain is still linear and is independent of the temperature.

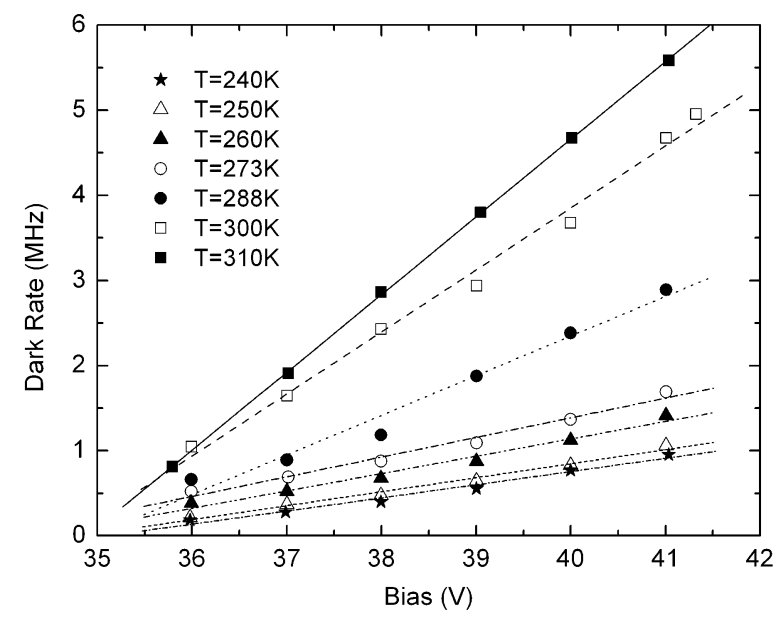

Fig. 9. Dark rate as a function of the bias voltage and of the temperature.

\section{Dark Count Rate}

Concerning the dark count, this was evaluated by a fast discriminator with a threshold set at half of the expected signal amplitude. The after-pulses with a height lower than this level have not been counted by the scaler. In these conditions, for a given temperature, the dark count rate increases linearly with respect to the bias voltage as shown in Fig. 9. This is due to the increase in electric field strength (in the multiplication area of the sensor) with the applied potential.

The dark count rate was measured as a function of the temperature while the gain was kept constant. This was achieved by changing the applied bias voltage in accordance to the values plotted in Fig. 7. The plot shapes obtained with our sample (which has a higher gain) are consistent with other published results [8]-[11], as reported in Fig. 10.

The highest gain and the lowest dark count rate of SiPM were recorded at the lowest temperature at which tests were performed (Fig. 11). As the requirements can differ dependent on the applications, it is often not practical to operate in a so low temperature range. The optimum operating temperature, at which the gain is maximum and the dark count minimum for different operating bias voltage, was found to be in the range

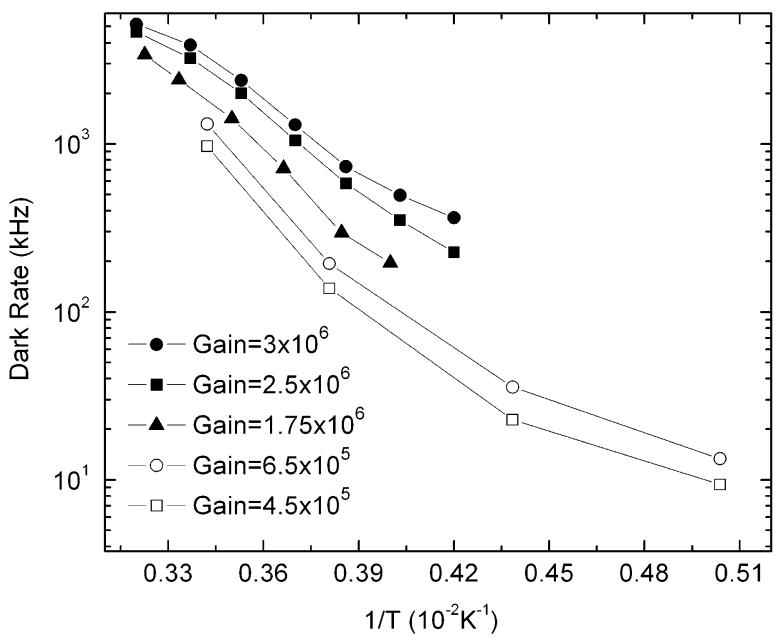

Fig. 10. Dark rate as a function of the temperature compared with experimental data from [8]. Note the difference between the gain of the samples.

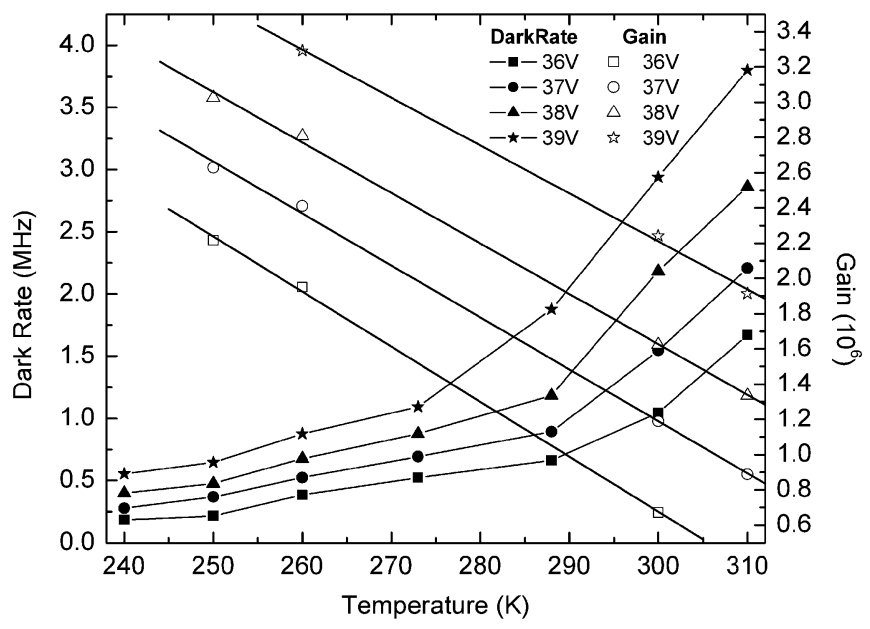

Fig. 11. Gain and dark rate as a function of the temperature.

290-295 K, corresponding, on the abscisse axis, at the interception points of the gain and dark rate plots at the same bias (Fig. 11). At temperatures above $295 \mathrm{~K}$ the dark count becomes very large leading to a high value of the dark rate. Further research on the device structure and architecture is under way to increase the maximum operating temperature of the detector while reducing the dark count rate.

\section{CONCLUSION}

In this paper a thermal and electrical characterization of SiPM has been reported. A variation of $2.1 \% / \mathrm{K}$ of the breakdown voltage has been estimated; this result indicates that some stringent applications need the use of a thermal regulator to stabilize the breakdown threshold and the noise level of the device. It was also found that the maximum operating temperature of the device is approximately $295 \mathrm{~K}$, since the SNR deteriorates with the increase of temperature, leading to an exponential increase of the dark rate and a linear decrease of the device gain.

Experiments on repeatability of temperature measurements and radiation hardness of SiPM for space and medical applications are under way. 


\section{ACKNOWLEDGMENT}

This work has been carried out in the framework of The DASiPM Collaboration which involves research groups from the Universities of Pisa, Bologna, Bari and Perugia. Also, the INFN/FBK-irst MEMS project's aim is the development of SiPMs for medical and space physics applications.

\section{REFERENCES}

[1] V. Golovin, "Novel type of avalanche photodetector with Geiger mode operation," Nucl. Instr. Meth., vol. A 518, pp. 60-564, 2004.

[2] C. Piemonte, "A new silicon photomultiplier structure for blue light detection," Nucl. Instr. Meth., vol. A 568, pp. 224-232, 2006.

[3] C. Piemonte, R. Battiston, M. Boscardin, G. F. Dalla Betta, A. Del Guerra, N. Dinu, A. Pozza, and N. Zorzi, "Characterization of the first prototypes of silicon photomultiplier fabricated at ITC-irst," IEEE Trans. Nucl. Sci., vol. 54, no. 1, pp. 236-244, Feb. 2007.

[4] N. Dinu, R. Battiston, M. Boscardin, G. Collazuol, F. Corsi, G. F. Dalla Betta, A. Del Guerra, G. Llosa, M. Ionica, G. Levi, S. Marcatili, C. Marzocca, C. Piemonte, G. U. Pignatel, A. Pozza, L. Quadrani, C. Sbarra, and N. Zorzi, "Development of the first prototypes of silicon photomultiplier (SiPM) at ITC-irst," Nucl. Instr. Methods Phys. Res., vol. A 572, pp. 422-426, 2007.
[5] M. Singh Tyagi, "Zener and avalanche breakdown in silicon alloyed p-n junctions-II," Solid-State Electron., vol. 11, pp. 117-128, 1968.

[6] K. G. McKay, "Avalanche breakdown in silicon," Phys. Rev., vol. 94, p. 877, 1954.

[7] S. M. Sze, Semiconductor Physics. New York: Wiley, 1981.

[8] G. Bondarenko, P. Buzhan, B. Dolgoshein, V. Golovin, E. Gushin, A. Ilyin, V. Kaplin, A. Karakash, R. Klanner, V. Pokachalov, E. Popova, and K. Smirnov, "Limited Geiger-mode microcell silicon photodiode: New results," Nucl. Instr. Meth., vol. A 442, pp. 187-192, 2000.

[9] P. Buzhan, B. Dolgoshein, L. Filatov, A. Ilyin, V. Kantzerov, V. Kaplin, A. Karakash, F. Kayumov, S. Klemin, E. Popova, and S. Smirnov, "Silicon photomultiplier and its possible applications," Nucl. Instr. Meth., vol. A 504, pp. 48-52, 2003.

[10] P. Buzhan, B. Dolgoshein, L. Filatov, A. Ilyin, V. Kaplin, A. Karakash, S. Klemin, R. Mirzoyan, A. N. Otte, E. Popova, V. Sosnovtsev, and M. Teshima, "Large area silicon photomultipliers: Performance and applications," Nucl. Instr. Meth., vol. A 567, pp. 78-82, 2006.

[11] N. Otte, B. Dolgoshein, J. Hose, S. Klemin, E. Lorenz, R. Mirzoyan, E. Popov, and M. Teshima, "The SiPM-A new photon detector for PET,” Nucl. Phys. B (Proc. Suppl.), vol. 150, pp. 417-420, 2006. 